\title{
Carregamento e caracterização do fármaco carboplatina na microestrutura microporosa do biomaterial granulado bifásico micro e nanoestruturado de fosfato de cálcio
}

\author{
Characterization of carboplatin loaded \\ micro-nanostructured porous granular \\ material in a biphasic calcium \\ phosphate ceramic system
}

Cristiane Savicki ${ }^{1}$, Nelson Heriberto Almeida Camargo ${ }^{1}$, Enori Gemelli ${ }^{1}$, Cleomar Copatti ${ }^{2}$, Nicolas Almeida Camargo ${ }^{3}$, Daiara Floriano Silva ${ }^{1}$

\footnotetext{
${ }^{1}$ Universidade do Estado de Santa Catarina, Centro de Ciências Tecnológicas, Departamento de Engenharia Mecânica Campus Universitário, Bairro Zona Industrial Norte, 89219-710, Joinville, Santa Catarina, Brasil. e-mail: cristianesavicki@gmail.com,dem2nhac@joinville.udesc.br, enori.gemelli@udesc.br, daiara.floriano@gmail.com ${ }^{2}$ Universidade do Estado de Santa Catarina, Centro de Ciências Tecnológicas, Departamento de Química, Joinville, Santa Catarina, Brasil.

e-mail: copattic@yahoo.com

${ }^{3}$ Universidade do Estado de Santa Catarina, Centro de Ciências Tecnológicas, Departamento de Física, Joinville, Santa Catarina, Brasil.

e-mail: nico.al.camargo@gmail.com
}

\begin{abstract}
RESUMO
As biocerâmicas bifásicas hidroxiapatita/fosfato tricálcico-beta (HA/TCP- $\beta$ ) microporosas são pesquisadas e se destacam em aplicações biomédicas como substitutos dos tecidos ósseos. Estes biomateriais são biocompatíveis e apresentam microestruturas microporosas interconectadas, que permitem sua utilização no reparo do tecido ósseo. Devido às suas características microestruturais, estes biomateriais vem sendo também avaliados para liberação controlada de fármacos. O presente trabalho teve como objetivo a incorporação do fármaco carboplatina no biomaterial granulado bifásico microporoso $60 \% \mathrm{HA} / 40 \% \mathrm{TCP}-\beta$ pelo método de alto vácuo e caracterização do biomaterial carregado, nas concentrações de $1,5,10$ e $20 \mathrm{mg} / \mathrm{g}$ de carboplatina no biomaterial. Os resultados apresentados envolvem a caracterização morfológica, microestrutural, cristalográfica e química do biomaterial granulado bifásico microporoso. A microscopia eletrônica de varredura (MEV) mostrou em suas micrografias a presença dos precipitados de carboplatina na microestrutura microporosa do biomaterial. O resultado da difratometria de raios X (DRX) permitiu a identificação dos picos representativos da fase cristalina da HA, TCP- $\beta$ e da carboplatina depois do carregamento. A análise por espectroscopia na região do infravermelho (FTIR) evidenciou os modos vibracionais relativos aos fosfatos de cálcio, $\mathrm{OH}^{-} \mathrm{e}$ $\mathrm{PO}_{4}{ }^{3-}$ e também Pt-N e $\mathrm{NH}_{3}$ da molécula da carboplatina. Os resultados obtidos demonstraram que a microporosidade da biocerâmica e o método de alto vácuo possibilitaram o carregamento da carboplatina na microestrutura do biomaterial granulado.
\end{abstract}

Palavras-chave: biocerâmicas bifásicas, microporosidade, carregamento de fármaco, carboplatina.

\section{ABSTRACT}

The hydroxyapatite/beta-tricalcium phosphate (HA/ $\beta$-TCP) bioceramics, has been reported to be attractive for biomedical applications, used as bone tissue substitute materials. These biomaterials exhibited excellent biocompatibility and interconnected microporous microstructure that provides suitable environments for a controlled drug delivery system in bone treatments. The characterization study of the biphasic granular material $60 \% \mathrm{HA} / 40 \% \beta$-TCP, was performed before and after loading the carboplatin using a high vacuum method. The drug was loaded at concentrations of $1 \mathrm{mg} / \mathrm{g}, 5 \mathrm{mg} / \mathrm{g}, 10 \mathrm{mg} / \mathrm{g}$ and $20 \mathrm{mg} / \mathrm{g}$ in a biphasic granular biomaterial. The morphological, microstructural, crystallographic and chemical characterization of the granulated biomaterial was investigated. SEM analysis showed structures identified as drugs precipitates on the structure of the biomaterial. The drug has been identified using X-ray technique, also. FTIR analysis 
indicated $\mathrm{OH}^{-}$and $\mathrm{PO}_{4}{ }^{3-}$ from calcium phosphates and $\mathrm{Pt}-\mathrm{N}$ and $\mathrm{NH}_{3}$ vibrational bands from carboplatin molecules. The results showed that the high vacuum method was efficient for the incorporation of carboplatin in micropore structure of the granular biomaterial.

Keywords: biphasic bioceramics, microporosity, drug delivery, carboplatin.

\section{INTRODUÇÃO}

As patologias musculoesqueléticas de origem oncológica são problemas crescentes e levam à perda de tecidos ósseos, pela proliferação de metástase na estrutura óssea. As biocerâmicas bifásicas microporosas de fosfatos de cálcio se mostram promissoras como biomateriais de substituição óssea e como matrizes potenciais para liberação controlada de fármacos nos tratamentos da estrutura óssea. [1]. Os tratamentos oncológicos e as deficiências motoras decorrentes dos mesmos geram altos custos estatais e privados, inibindo boa parte da sociedade ao tratamento adequado, principalmente no setor público. Isso justifica o interesse nos âmbitos científico, industrial, comercial e governamental, que dedicam esforços e procuram inovar os métodos, as técnicas de diagnóstico e de tratamento, a fim de oferecer uma melhor expectativa de vida à população e reduzir os custos de tratamento [2]. Existe uma expectativa do setor público e privado no desenvolvimento de biomateriais inovadores, que possam liberar as drogas de forma controlada, reduzir efeitos colaterais de tratamentos oncológicos e reparar os tecidos ósseos, com custo acessível a uma larga faixa da população[3].

Os fosfatos de cálcio nanoestruturados são formados por micro e nanoestruturas microporosas interconectadas, com características físicas, químicas e mecânicas similares ao tecido ósseo humano [4-6]. Dentre estas biocerâmicas de fosfatos de cálcio, as mais pesquisadas são a hidroxiapatita, fosfato tricálcico- $\beta$ e composições bifásicas. Isso se deve por esses biomateriais apresentarem características físicas, micro e nanoestruturais favoráveis ao carregamento de fármaco, proteínas e a neoformação e reconstrução do tecido ósseo [710]. As biocerâmicas de fosfatos de cálcio micro e nanoestruturadas microporosas se revelam promissoras como sistemas de entrega localizado de medicamentos de forma controlada em tratamentos da estrutura óssea, por serem biodegradáveis quando aplicadas em meio biológico [1,9]. As pesquisas revelam que as biocerâmicas de fosfatos de cálcio microporosas são potenciais como matriz de liberação controlada de drogas em prevenção e tratamento de patologias como metástase óssea, osteomielite e osteoporose [3,11-13].

As biocerâmicas micro e nanoestruturadas de fosfatos de cálcio são uma inovação tecnológica e demonstram desempenhar a função de entrega localizada da droga, atingindo locais específicos do corpo. Essas biocerâmica podem contribuir com as terapias clínicas, reduzir os efeitos colaterais dos medicamentos e maximizar os efeitos farmacológicos $[1,9,10]$. Na oncologia, diversos estudos são realizados com o uso de biomateriais nanoestruturado de fosfatos de cálcio em tratamento de tumores na estrutura óssea. Os resultados mostram que esses biomateriais apresentam comportamento eficiente na entrega de fármacos in situ [14-17].

Este trabalho teve como objetivo o carregamento de carboplatina na microestrutura microporosa do biomaterial granulado bifásico 60\% HA/40\% TCP- $\beta$ e caracterização do biomaterial, antes e depois da incorporação do fármaco. A carboplatina foi incorporada nas concentrações de 1, 5, 10 e $20 \mathrm{mg}$ por grama de biomaterial, com o uso do método de alto vácuo. O objetivo foi a fixação do fármaco na superfície e no interior da microporosidade do biomaterial. A carboplatina é um fármaco utilizado em tratamentos quimioterápicos e pertence à segunda geração de drogas derivadas da platina. Quimicamente a molécula da carboplatina, ou cis-diamino(1,1-ciclobutanodicarboxilato)platina (II) é representada pela estrutura molecular mostrada na Figura $1[18]$.

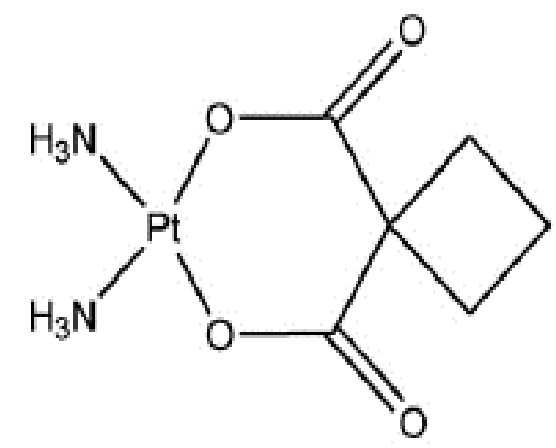

Figura 1: Estrutura molecular da carboplatina.

Estudos anteriores de caracterização de biomaterial granulado bifásico 60\% HA/40\% TCP- $\beta$ carregado com carboplatina na dose de $70 \mathrm{mg} / \mathrm{g}$ pelo método de alto vácuo, realizados por outros autores [19], não 
demostraram alterações microestruturais e químicas no biomaterial ou no fármaco. No presente trabalho foram realizados os estudos de caracterização morfológica, microestrutural, cristalográfica e química da carboplatina e dos biomateriais micro e nanoestruturados, antes e depois da incorporação do fármaco.

\section{MATERIAIS E MÉTODOS}

O material granulado bifásico microporoso foi fornecido pelo Grupo de Biomateriais da UDESC, esse foi fabricado conforme descrito pelos autores em publicações anteriores [20, 21]. O biomaterial sinterizado a $1100{ }^{\circ} \mathrm{C} / 2 \mathrm{~h}$ se apresentou na forma de grânulos microporos com tamanhos inferiores a $500 \mu \mathrm{m}$ e superiores a $200 \mu \mathrm{m}$.

A carboplatina utilizada foi a especialidade farmacêutica Tevacarbo, na concentração de $10 \mathrm{mg} / \mathrm{mL}$ em suspensão contendo manitol e água destilada. O manitol é um excipiente largamente utilizado em preparações farmacêuticas, de fórmula $\mathrm{C}_{6} \mathrm{H}_{14} \mathrm{O}_{6}$, e denominação hexano-1,2,3,4,5,6-hexol e possui estrutura molecular indicada na Figura 2 [22].

Figura 2: Estrutura molecular do D-manitol.

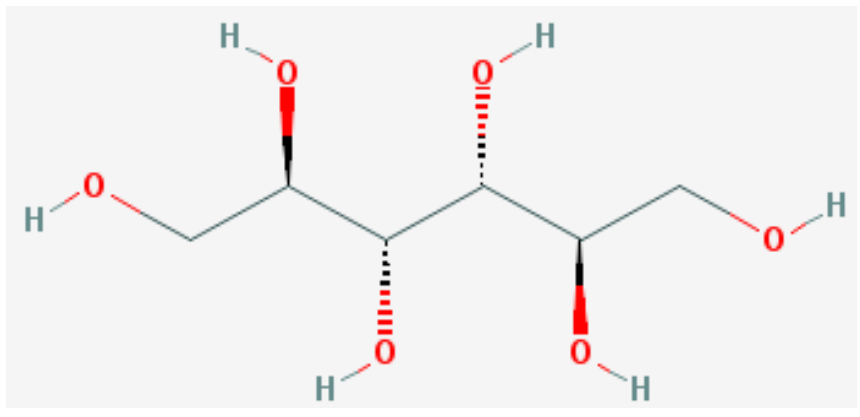

O fármaco foi incorporado nas concentrações de 1, 5, 10 e $20 \mathrm{mg}$ de carboplatina por grama de biomaterial. O método de alto vácuo foi utilizado para carregamento do fármaco ao biomaterial. O biomaterial granulado foi imerso na solução e submetido a vácuo de $10^{-3}$ mbar até evaporação completa do solvente, restando somente o biomaterial com os precipitados de carboplatina e manitol em sua microestrutura.

Os estudos de caracterização foram realizados sobre o pó de carboplatina e do biomaterial granulado bifásico. A caracterização morfológica e microestrutural foi realizada em um microscópio eletrônico de varredura, com efeito de Campo, marca JEOL, modelo JSM-6701F, no sistema de elétrons secundários (SE), tensão de $15 \mathrm{KV}$ e corrente de aceleração de $10 \mu \mathrm{A}$. A técnica de difratometria de raios X forneceu a cristalografia da carboplatina e do biomaterial. As análises foram realizadas em um equipamento marca Shimadzu XRD-600, utilizando anti-catodo de tubo de cobre $\operatorname{com} \lambda=1,54060 \AA ̊$, $40 \mathrm{kV}$ de tensão e $30 \mathrm{~mA}$ de intensidade de corrente e deslocamento $2 \theta$ de $2 \%$ min. Os resultados obtidos foram comparados com dados do Joint Committee on Powder Diffraction Standards (JCPD). As análises de espectroscopia na região do infravermelho (FTIR) foram realizadas em espectrômetro marca Pelkin Elmer com refletância atenuada e intervalo de análise de 4000 a $450 \mathrm{~cm}^{-1}$.

\section{RESULTADOS}

\subsection{Caracterização do biomaterial bifásico HA/TCP- $\beta$}

O resultado obtido pela microscopia eletrônica de varredura sobre o biomaterial granulado bifásico, revelou em suas micrografias (Figura 3a) uma morfologia irregular dos grânulos, com uma microestrutura microporosa interconectada formada por micro e nano grãos, conforme representado pelas setas brancas os micro grãos e pelas azuis os nano grãos (Figura 3b). 

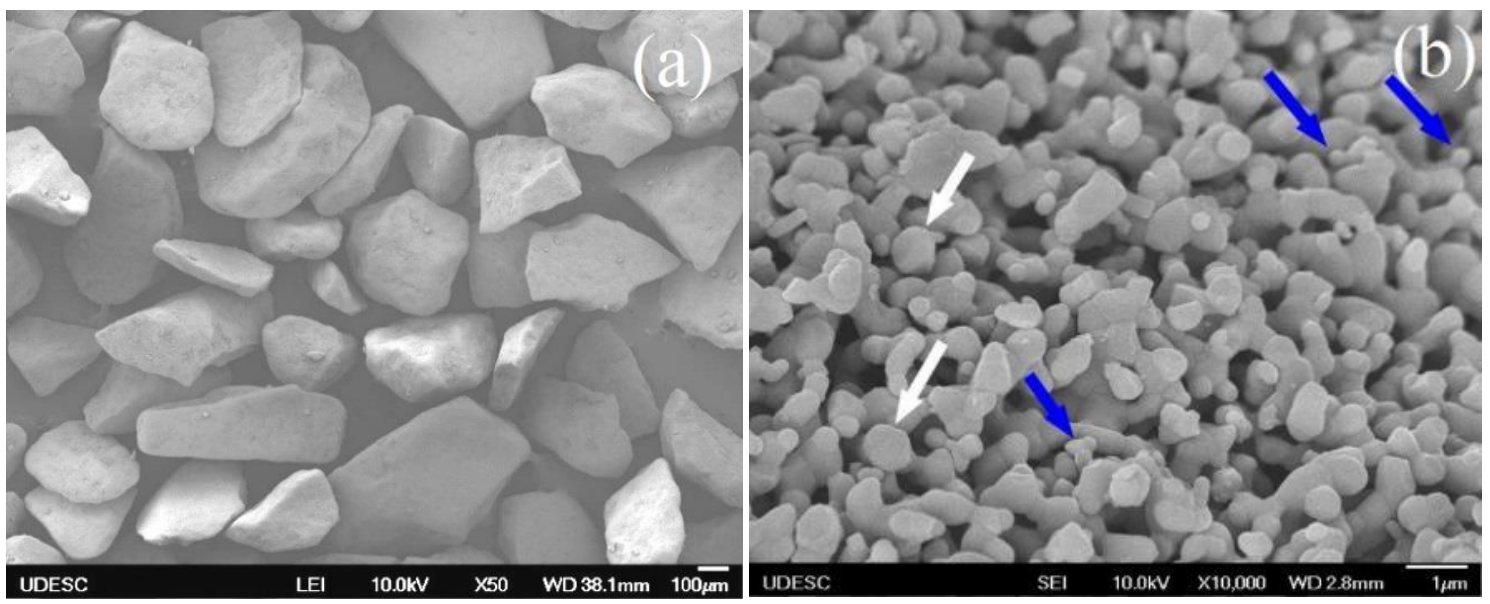

Figura 3: Micrografia mostrando a morfologia do biomaterial granulado bifásico HA/TCP- $\beta$, sinterizado a $1100{ }^{\circ} \mathrm{C} / 2 \mathrm{~h}$ (a) e a microestrutura (seta na cor branca) e nanoestrutura de grãos do biomaterial (seta na cor azul) (b)

A análise de difratometria de raios $\mathrm{X}$, obtida sobre o biomaterial granulado bifásico micro e nanoestruturado evidenciou em seu difratograma (Figura 4) a presença da fase cristalina da HA e TCP- $\beta$, com sistema cristalino hexagonal com plano principal de difração (211) para a HA e sistema cristalino romboédrico, com plano principal de difração (0210) para o TCP- $\beta$.

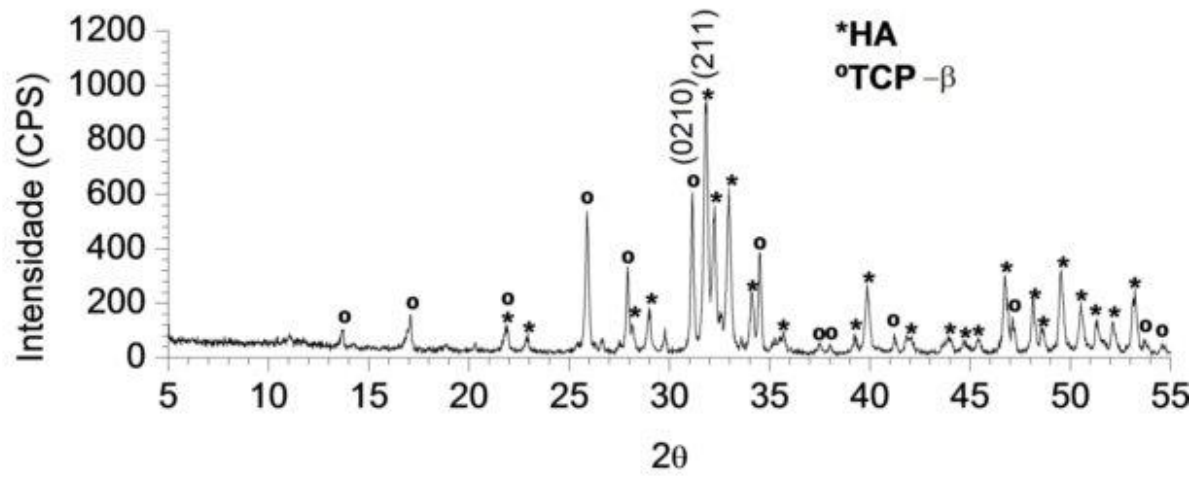

Figura 4: Difratograma de raios X obtido sobre o biomaterial granulado bifásico HA/TCP- $\beta$.

O espectro da região do infravermelho do biomaterial granulado bifásico revelou (Figura 5) os modos vibracionais do grupamento $\mathrm{OH}^{-}$, representativos da hidroxila presente no biomaterial granulado, associado a HA em 3572 e $633 \mathrm{~cm}^{-1}$. Em relação ao grupamento $\mathrm{PO}_{4}{ }^{3-}$ foram identificados os modos vibracionais em 1088 e $1025 \mathrm{~cm}^{-1}$, correspondentes ao estiramento assimétrico P-O (v3); já em $962 \mathrm{~cm}^{-1}$ o modo correspondente ao estiramento simétrico P-O (v1) e em 603 e 570 os modos de dobramento angular assimétrico O-P-O ( $v 4)$, conforme já observado por outros autores [23-25].

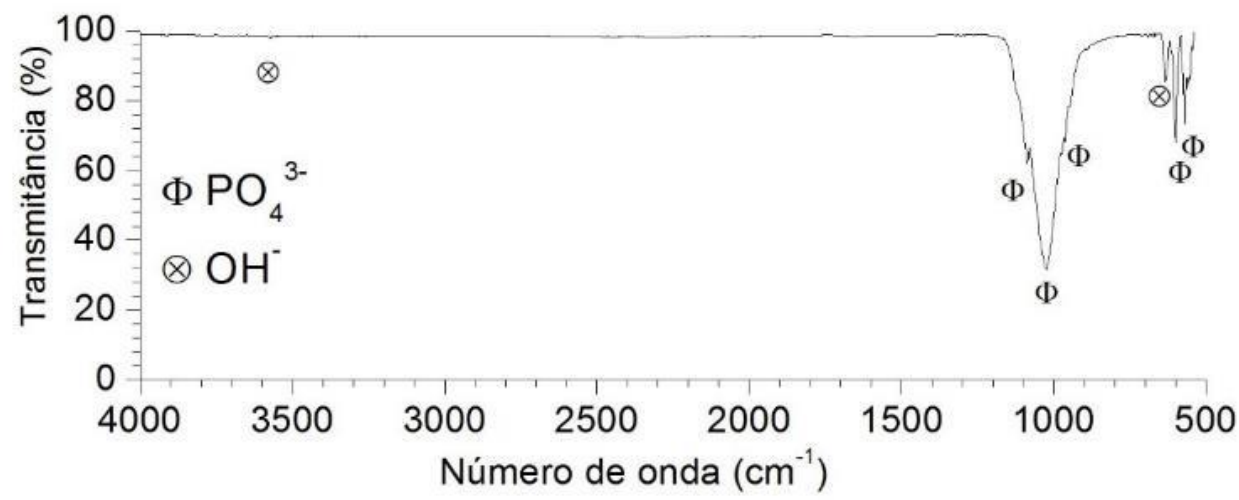

Figura 5: Espectro na região do infravermelho obtido sobre o biomaterial granulado bifásico HA/TCP- $\beta$. 


\subsection{Caracterização do fármaco carboplatina/manitol}

As figuras $6 \mathrm{a}$ e $6 \mathrm{~b}$ mostram a morfologia do fármaco utilizado para o carregamento, representado por finos cristais presentes nas micrografias, na cor clara, indicativos da presença da carboplatina, e pelos precipitados escuros atribuídos ao manitol, de morfologia semelhante a verificada por outros autores [26]. O manitol se encontra presente no fármaco por ter sido utilizado como elemento matricial para fixação da carboplatina.
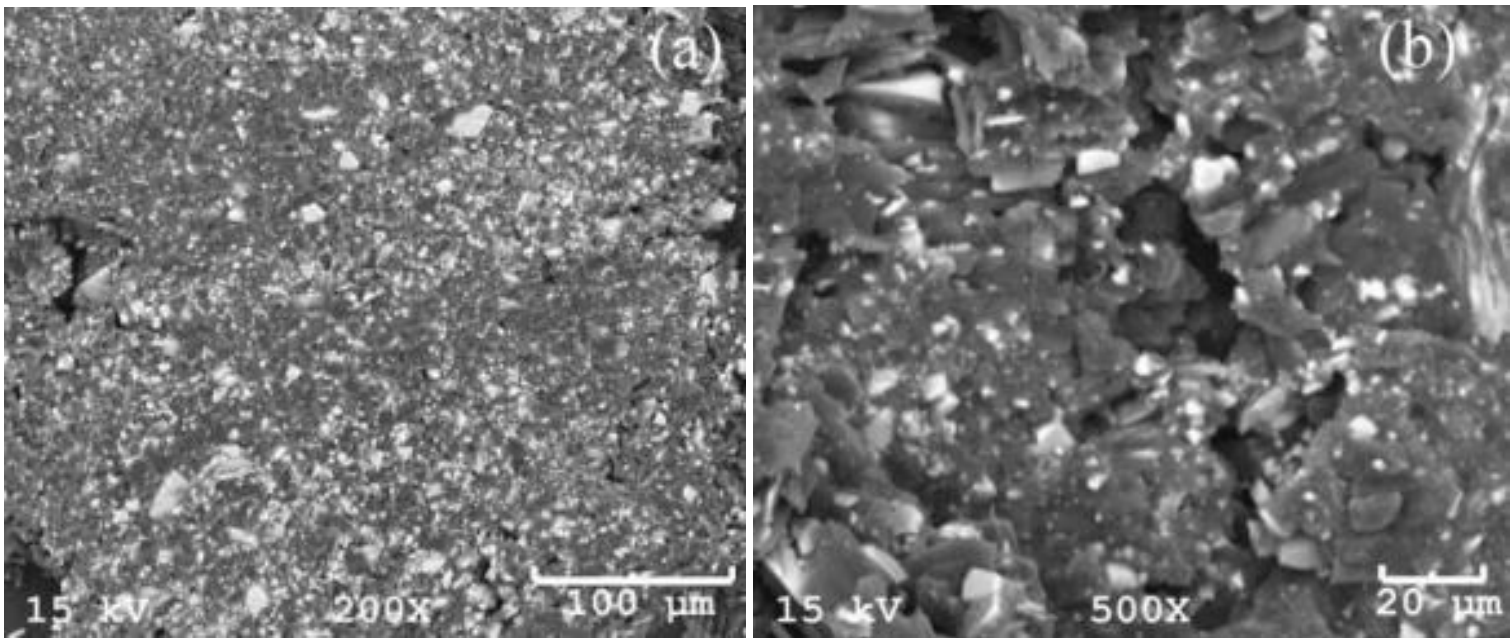

Figura 6: Micrografias obtidas sobre a carboplatina/manitol (a) e (b)

O resultado da caracterização cristalográfica obtido sobre a carboplatina revelou em seu difratograma de raios X (Figura 7) os picos representativos da fase cristalina da carboplatina (Pt) e picos de baixa intensidade representativo do manitol presente no fármaco.

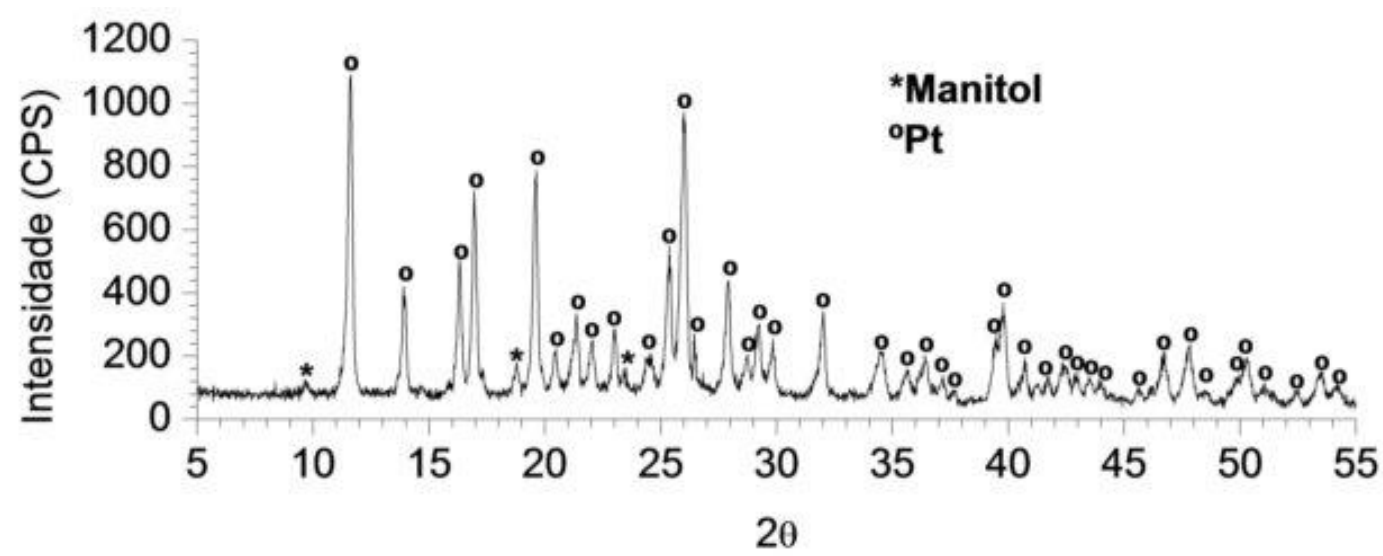

Figura 7: Difratograma de raios X obtido sobre a o fármaco carboplatina contendo a matriz manitol.

O resultado obtido sobre uma amostra de manitol revelou em seu espectro na região do infravermelho modos vibracionais próprios da sua molécula. A Figura 8a mostra o espectro obtido sobre o manitol, onde se observa os modos vibracionais em 3393 e $3282 \mathrm{~cm}^{-1}$ correspondentes aos estiramentos dos grupos funcionais hidroxila. Em $2985 \mathrm{~cm}^{-1}$, verifica-se o modo vibracional de estiramento de fraca intensidade referente ao grupamento $-\mathrm{CH}$, em $1418 \mathrm{~cm}^{-1}$ o modo vibracional referente ao dobramento angular simétrico no plano do grupamento $-\mathrm{CH}_{2}$ e em $1281 \mathrm{~cm}^{-1}$ o modo vibracional de dobramento angular no plano do grupo hidroxila. Também se constata no espectro os modos vibracionais de forte intensidade em 1078 e $1018 \mathrm{~cm}^{-1}$, que estão associados à ligação $\mathrm{C}-\mathrm{O}$ dos estiramentos da primeira e segunda hidroxila da molécula do manitol, que é um poliálcool (ver Figura 2). Entre 929 e $629 \mathrm{~cm}^{-1}$ têm a ocorrência de bandas de baixa intensidade, denominada fingerprint region, que é um conjunto de bandas que não têm necessariamente atribuições específicas de grupos funcionais, mas formam um padrão característico, podendo assim auxiliar na identificação de compostos. Muitas substâncias apresentam a chamada fingerprint region, que no manitol é localizada entre 
aproximadamente 930 a $600 \mathrm{~cm}^{-1}$ (na Figura 8, as principais bandas desta região estão indicadas em suas posições, em $\mathrm{cm}^{-1}$ ). Esses modos vibracionais também já foram descritos por outros autores [26] em estudo de caracterização do D-manitol.

O resultado obtido sobre o fármaco revelou em seu espectro na região do infravermelho (Figura 8b) os principais modos vibracionais representativos da molécula da carboplatina e manitol. Constatam-se os modos vibracionais associados ao grupo funcional de amônia em $3189 \mathrm{~cm}^{-1}$, referente ao estiramento N-H, associado ao grupamento $\mathrm{NH}_{3}$ da carboplatina, adjacente à banda larga de modo vibracional de estiramento das hidroxilas do manitol. O grupo amônia também pode ser identificado nos modos vibracionais em $1637 \mathrm{~cm}^{-1} \mathrm{e} \mathrm{em}$ $765 \mathrm{~cm}^{-1}$. Observa-se ainda os modos vibracionais referentes ao grupamento $-\mathrm{CH}_{2}$ em 2960, 1462 e $1249 \mathrm{~cm}^{-}$ ${ }^{1}$ relacionados a carboplatina e o manitol presentes no fármaco. Também pode ser verificado o modo vibracional de estiramento em $1608 \mathrm{~cm}^{-1}$ relacionado às ligações $\mathrm{C}=\mathrm{O}$ associadas ao grupo funcional do ácido carboxílico presente na carboplatina. Em 1380 e $1348 \mathrm{~cm}^{-1}$ ocorrem os modos vibracionais de estiramento das ligações simples $\mathrm{C}-\mathrm{O}$ da molécula de carboplatina, enquanto que modos vibracionais de intensidade superior de ligações C-O em 1084 e $1022 \mathrm{~cm}^{-1}$ podem estar associados à molécula do manitol. Os modos localizados na região do espectro compreendida entre 930 a $623 \mathrm{~cm}^{-1}$ corresponde à fingerprint region do manitol, já identificada anteriormente na análise do manitol puro (Figura 8a) e também verificada na análise da carboplatina com manitol (Figura 8b) [27,28].

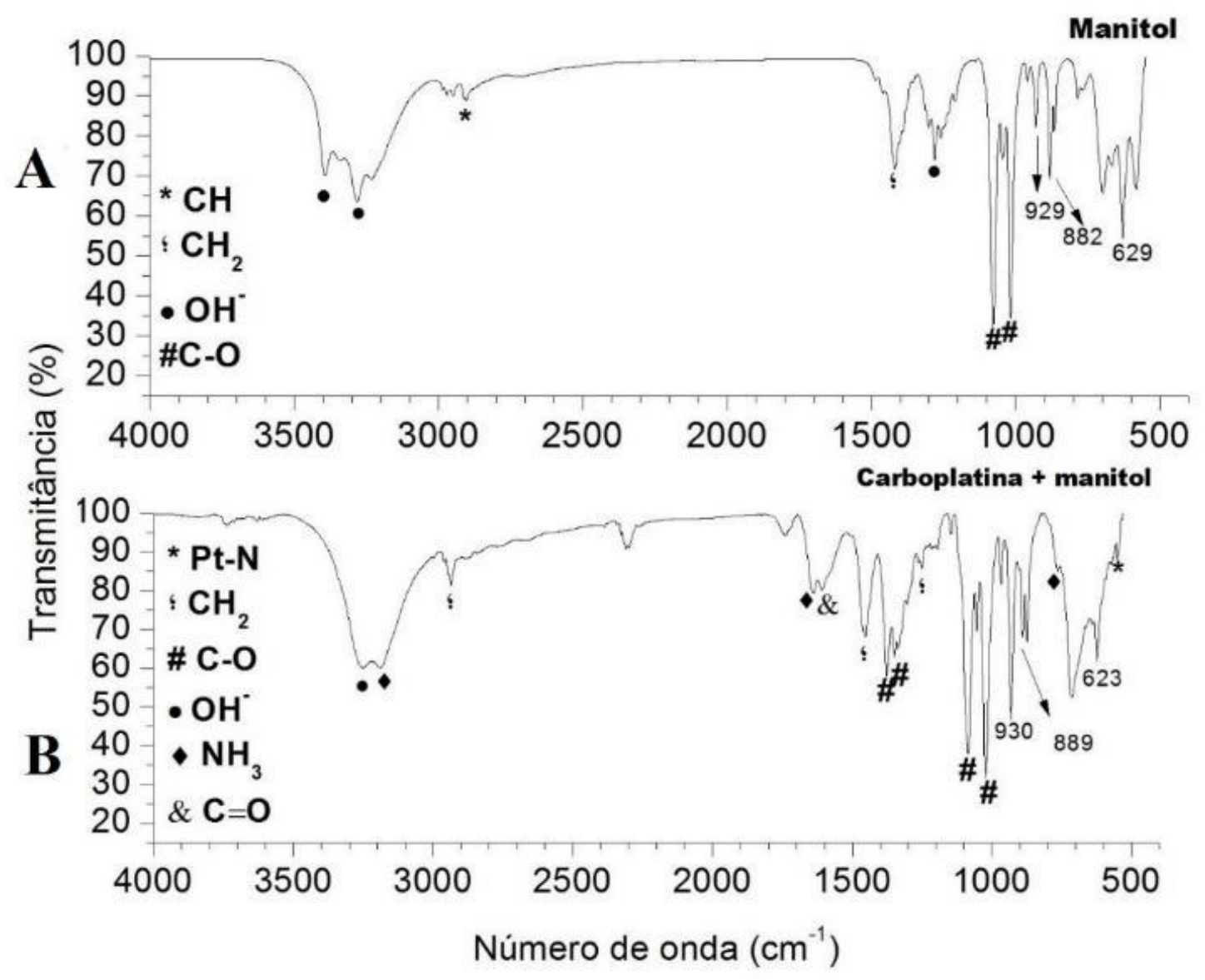

Figura 8: Espectros na região do infravermelho obtidos sobre o manitol puro e do fármaco carboplatina + manitol.

Os modos vibracionais representativos da molécula de carboplatina se referem às ligações Pt-N e PtO. O modo vibracional representativo da ligação Pt-N é normalmente encontrado em aproximadamente 545 $\mathrm{cm}^{-1}$, que indica a presença da carboplatina, conforme foi observado nesse trabalho e por outros autores [27]. Já os modos vibracionais representativos da ligação Pt-O de alta intensidade se encontram em aproximadamente 350 e $475 \mathrm{~cm}^{-1}$, sendo que, o número de onda em $350 \mathrm{~cm}^{-1}$ não foi representado no espectro, em razão da análise ter sido realizada até $450 \mathrm{~cm}^{-1}$. 


\subsection{Caracterização do biomaterial carregado com carboplatina}

As figuras 9a, 9b, 9c e 9d mostram as micrografias obtidas sobre os biomateriais granulados bifásico nanoestruturados depois do carregamento do fármaco nas concentrações de $1,5,10$ e $20 \mathrm{mg} / \mathrm{g}$ de biomaterial, respectivamente.

As setas na cor preta sobre as micrografias mostram os precipitados nanométricos de carboplatina, na superfície de grãos do biomaterial granulado bifásico. Observou-se maior presença de precipitados para o biomaterial com a concentração de $20 \mathrm{mg} / \mathrm{g}$ (Figura 9d).
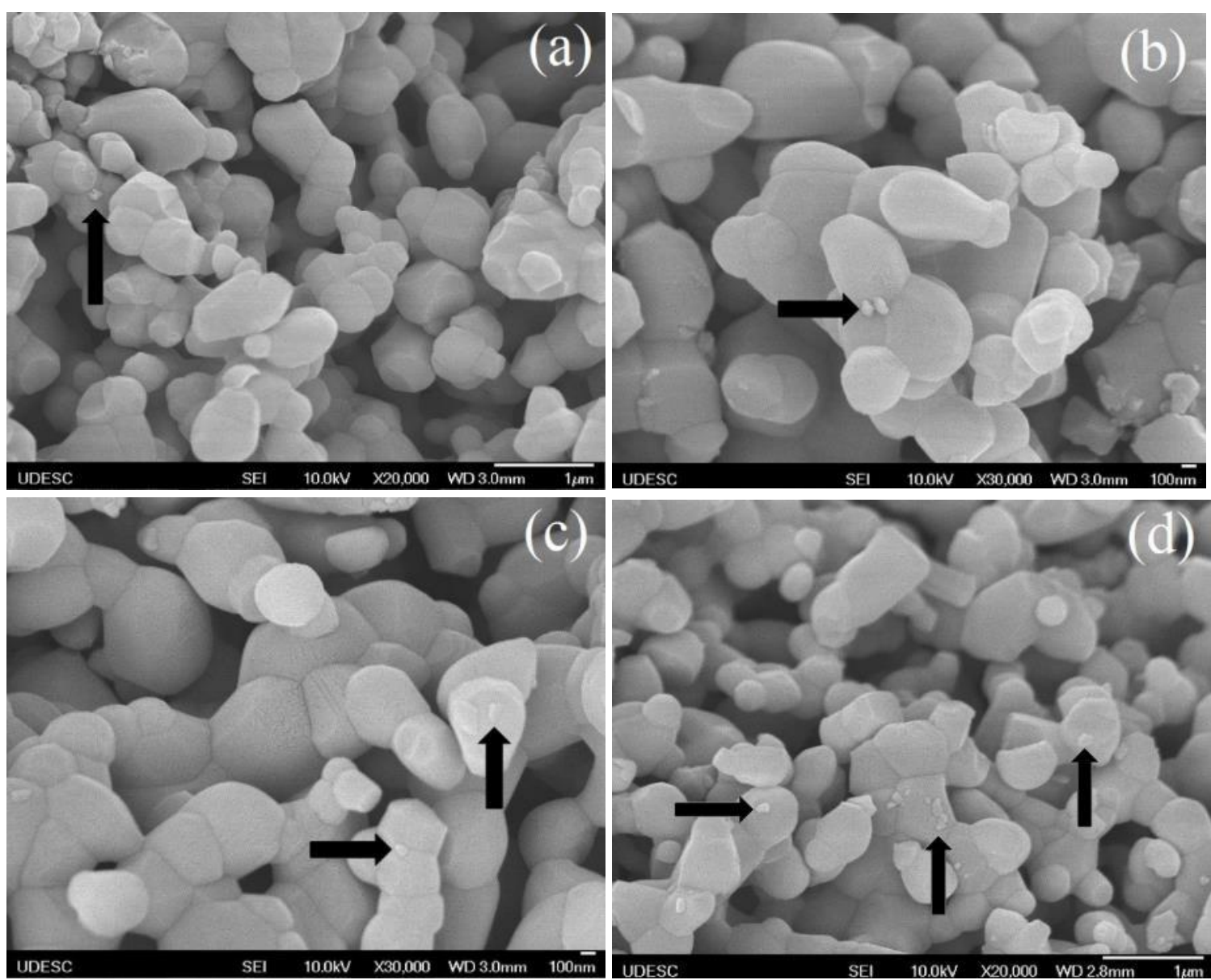

Figura 9: Micrografias mostrando a presença de carboplatina na superfície de grãos do biomaterial bifásico com $1 \mathrm{mg} / \mathrm{g}$ (a); $5 \mathrm{mg} / \mathrm{g}$ (b); $10 \mathrm{mg} / \mathrm{g}$ (c) e $20 \mathrm{mg} / \mathrm{g}$ (d).

A caracterização por difratometria de raios X, obtida sobre o biomaterial granulado bifásico micro e nanoestruturado na concentração de fármaco de $1 \mathrm{mg} / \mathrm{g}$ de biomaterial, não revelou em seu difratograma de raios $\mathrm{X}$ a presença de picos de difração representativos da fase cristalina da carboplatina (Figura 10a). Isto pode ser explicado pela pequena concentração de fármaco incorporado ao biomaterial. Já para as amostras contendo 5, 10 e $20 \mathrm{mg} / \mathrm{g}$, foram evidenciados picos representativos da fase cristalina do biomaterial granulado bifásico e da carboplatina (Figura 10b, 10c e 10d). Também se constata nos difratogramas de raios $\mathrm{X}$ (Figura 10b, 10c e 10d), um leve aumento da intensidade dos picos representativos da fase cristalina da carboplatina, o que está associado ao aumento da concentração do fármaco ao biomaterial. Outra observação foi a redução da intensidade dos picos representativos da fase cristalina do biomaterial granulado bifásico, se comparar os difratogramas com o obtido do biomaterial granulado bifásico sem fármaco (Figura 4). Essa redução da intensidade dos picos de difração pode estar relacionada à presença da fase cristalina da carboplatina e manitol na superfície de grãos e microporos do biomaterial. A presenças desses elementos pode ter modificado a superfície de grãos e microporos, inibindo a revelação dos planos de difração do biomaterial bifásico. 


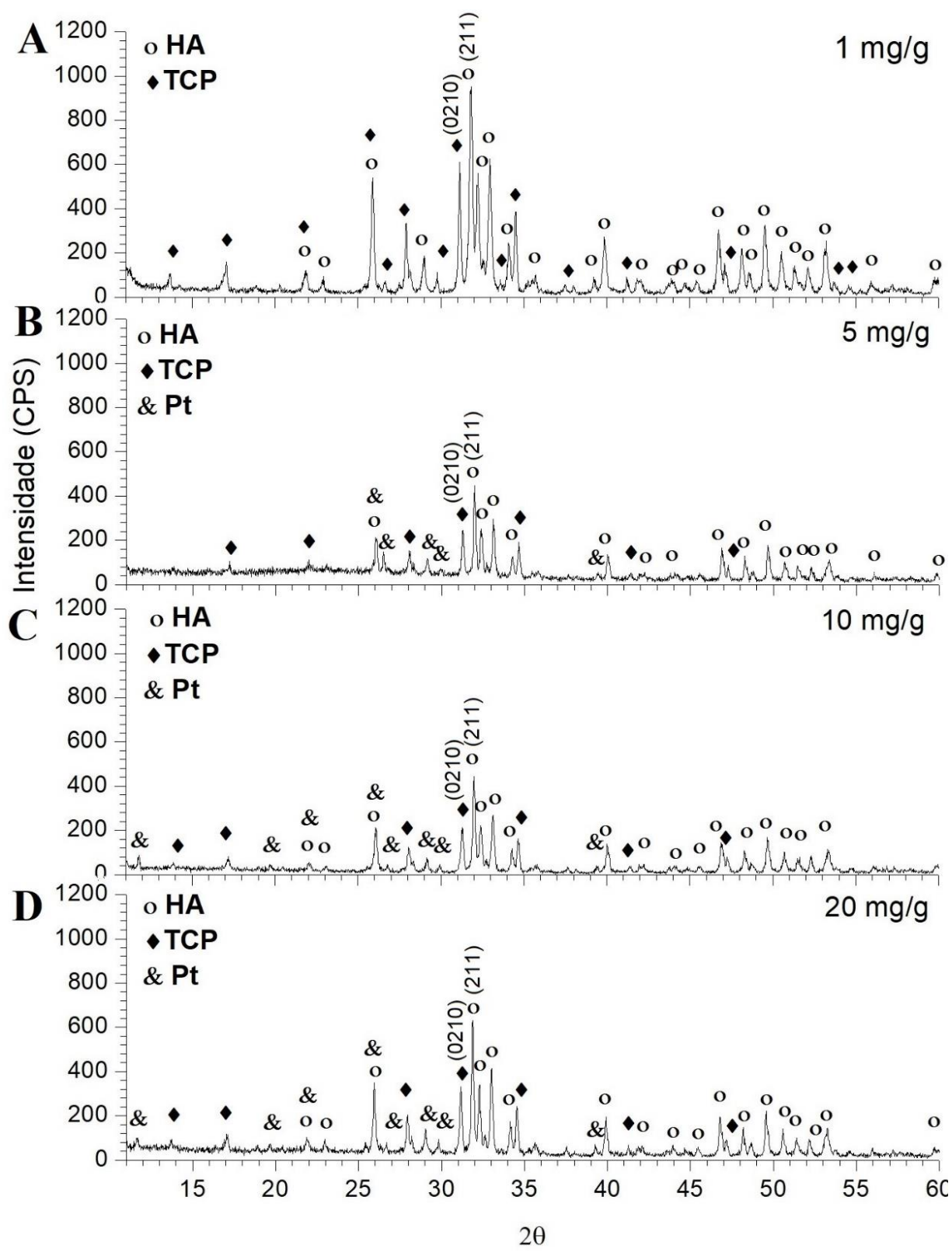

Figura 10: Difratogramas de raios $X$ obtidos sobre os biomateriais granulados bifásicos com $1 \mathrm{mg} / \mathrm{g}$ (a); $5 \mathrm{mg} / \mathrm{g}$ (b); 10 $\mathrm{mg} / \mathrm{g}$ (c) e $20 \mathrm{mg} / \mathrm{g}$ (d) de carboplatina.

Os espectros na região do infravermelho, obtidos sobre o biomaterial granulado bifásico micro e nanoestruturado, depois de carregamento com o fármaco, revelaram os modos vibracionais característicos para o biomaterial de HA/TCP- $\beta$ antes do carregamento, não tendo sido evidenciadas alterações nas posições dos modos vibracionais dos grupamentos $\mathrm{OH}^{-}$em 3572 e $633 \mathrm{~cm}^{-1}$ e grupamentos de fosfatos. Foram identificados os modos vibracionais em 1088 e $1025 \mathrm{~cm}^{-1}$, correspondentes ao estiramento assimétrico P-O (v3); em $962 \mathrm{~cm}^{-1}$ o estiramento simétrico P-O (v1), com baixa intensidade e em 607 e $570 \mathrm{~cm}^{-1}$ os modos vibracionais correspondentes aos modos de dobramento angular assimétrico O-P-O (v4), conforme já observado por outros autores (Figura 11) [23-25]. Não foram identificados modos vibracionais característicos da carboplatina e do manitol na região do espectro em torno de $1000 \mathrm{~cm}^{-1}$, possivelmente pela sobreposição destes modos relacionados aos grupamentos fosfatos de alta intensidade, devido à baixa concentração do fármaco ao biomaterial. Entretanto, constatou-se uma alteração significativa nos espectros de infravermelho, na região próxima a $500 \mathrm{~cm}^{-1}$, comportamento verificado para todas as concentrações de fármaco ao biomaterial granulado bifásico. Essa alteração pode ser atribuída à sobreposição de modos vibracionais dos grupamentos dos fosfatos de menor intensidade com os modos vibracionais dos grupamentos da molécula de carboplatina e/ou do manitol, nesta região do espectro. Para os biomateriais carregados com 10 e $20 \mathrm{mg} / \mathrm{g}$, ficaram evidenciados os modos vibracionais de fraca transmitância em aproximadamente 3290 e $3200 \mathrm{~cm}^{-1}$, relativos ao estiramen- 
to assimétrico do grupamento $\mathrm{NH}_{3}$ da carboplatina. A presença do modo vibracional em aproximadamente $535 \mathrm{~cm}^{-1}$ está associada à ligação Pt-N. Esses resultados mostram a presença do fármaco na microestrutura do biomaterial granulado bifásico. Essas observações já foram verificadas por outros autores que efetuaram o carregamento de carboplatina em biomateriais bifásicos [19].

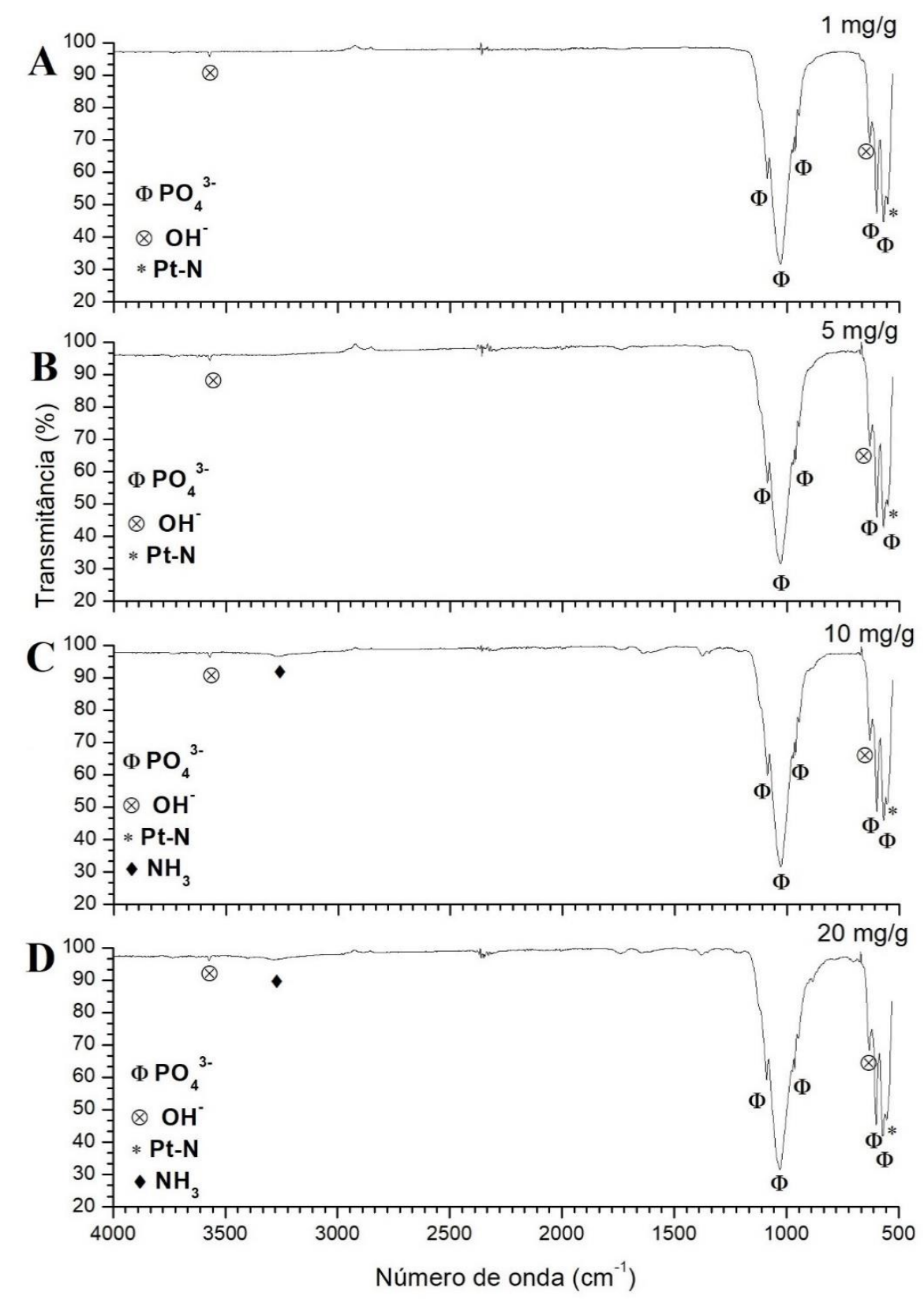

Figura 11: Espectro na região do infravermelho obtidos sobre os biomateriais granulados bifásicos com $1 \mathrm{mg} / \mathrm{g}$ (a); 5 $\mathrm{mg} / \mathrm{g}$ (b); $10 \mathrm{mg} / \mathrm{g}$ (c) e $20 \mathrm{mg} / \mathrm{g}$ (d) de carboplatina.

\section{DISCUSSÃO}

A matriz de fosfato de cálcio bifásica $60 \% \mathrm{HA} / 40 \% \mathrm{TCP}-\beta$, fornecida pelo Grupo de Biomateriais da UDESC para realização desse trabalho, se apresentou com microestrutura microporosa interconectada formada por micro e nano grãos. Essas características microestruturais são favoráveis a molhabilidade, a capilaridade e adesão de células de osteoblastos na superfície de grãos e microporos $[8,28]$. Resultados de estudos in vivo, realizados por diferentes autores, mostraram que essas características também contribuíram com os processos de osseointegração e neoformação óssea [7, 29, 30]. As biocerâmicas de fosfatos de cálcio microporosas, formadas por micro e nano grãos, com microestrutura microporosa interconectada, se apresentam potenciais como matriz de carregamento de fármacos e proteínas em suas microestruturas microporosas, conforme estudos 
já realizados por alguns autores [11-16, 31, 32].

As fases cristalinas identificadas pela difratometria de raios $\mathrm{X}$ sobre o biomaterial granulado bifásico micro e nanoestruturado foram hidroxiapatita e fosfato tricálcico- $\beta$, mostrando que o biomaterial é bifásico. Os resultados encontrados pela difratometria de raios $\mathrm{X}$, sobre os biomateriais granulados bifásicos carregado com o fármaco, mostrou a presença da fase cristalina dos precipitados de carboplatina presentes na superfície de grãos e microporos do biomaterial bifásico a partir da dose de $5 \mathrm{mg} / \mathrm{g}$, observando o aumento da intensidade dos picos de difração da fase cristalina de carboplatina, conforme se aumento a dose carregada. Outra observação foi a redução da intensidade dos picos representativos das fases cristalinas do biomaterial bifásico granulado (Figuras 4 e 10), que pode estar relacionado à modificação superficial dos grãos do biomaterial bifásico quando em presença da carboplatina.

A espectroscopia na região do infravermelho, obtida sobre o biomaterial granulado bifásico micro e nanoestruturado, antes e após o carregamento, revelou em seu espectro os modos vibracionais típicos dos grupamentos $\mathrm{PO}_{4}{ }^{3-}$ e $\mathrm{OH}^{-}$característicos dos fosfatos de cálcio (Figuras 5 e 11). Observou-se o modo vibracional em torno $3500 \mathrm{~cm}^{-1}$ representativo do grupamento $\mathrm{OH}^{-}$, relacionado a hidroxiapatita, os modos de estiramento $v 3$ entre 1090 e $1020 \mathrm{~cm}^{-1}$ e em $962 \mathrm{~cm}^{-1}$, o estiramento $v 1$ relacionados às ligações P-O dos fosfatos de cálcio; em 603 e $570 \mathrm{~cm}^{-1}$ os modos vibracionais $v 4$ de dobramento angular O-P-O dos fosfatos. Em 633 $\mathrm{cm}^{-1}$ o modo vibracional do grupamento $\mathrm{OH}^{-}$indicativo da hidroxiapatita, caso já observado em outros estudos [23-25]. Em relação aos biomateriais carregados, as baixas doses carregadas (1 a $20 \mathrm{mg} / \mathrm{g}$ ) não permitiram evidenciar todos os modos vibracionais identificados na análise do fármaco. A baixa intensidade de transmitância da carboplatina e manitol nos biomateriais, após carregamento, é devida às baixas doses carregadas e também à sobreposição de modos vibracionais típicos do biomaterial, carboplatina e manitol, que ocorrem em regiões similares no espectro. Os principais modos vibracionais dos biomateriais ocorrem nas regiões em torno de $3500 \mathrm{~cm}^{-1}$, entre aproximadamente 1100 a $1000 \mathrm{~cm}^{-1}$ e finalmente em 550 a $600 \mathrm{~cm}^{-1}$. Para a carboplatina e manitol, os principais modos vibracionais também foram verificados nestas regiões do espectro, não permitindo a evidenciação de todos os modos quando em presença do biomaterial. Por exemplo, os modos de estiramento das ligações C-O em 1084 e $1022 \mathrm{~cm}^{-1}$ foram evidenciados na análise da carboplatina com manitol, mas não foram verificados na análise do biomaterial carregado em virtude da localização na mesma região do espectro do modo vibracional de forte intensidade do estiramento $v 3$ do $\mathrm{PO}_{4}{ }^{3-}$. Entretanto, a técnica de FTIR permitiu a identificação do grupamento $\mathrm{NH}_{3}$ a partir de concentrações com $10 \mathrm{mg} / \mathrm{g} \mathrm{em}$ 3290 e $3200 \mathrm{~cm}^{-1}$ e a modificação da intensidade de transmitância para os modos vibracionais em aproximadamente 550 a $500 \mathrm{~cm}^{-1}$, resultados já verificados em outros trabalhos [19]. Também foi identificado o estiramento da ligação Pt-N em $500 \mathrm{~cm}^{-1}$, porém com baixa intensidade, para todas as concentrações. A baixa intensidade do modo vibracional de estiramento $\mathrm{Pt}-\mathrm{N}$ já foi verificada na análise da carboplatina em presença do manitol. Isto pode ser devido à região de localização da banda, próximo ao fim do espectro e na região das bandas de alta absorção dos modos de dobramento angular (v2) dos grupos fosfato.

Os resultados das caracterizações por MEV, DRX e FTIR realizadas no biomaterial após o carregamento evidenciaram a presença do fármaco na matriz fosfato de cálcio. As técnicas associadas ao uso de vácuo têm sido descritas como eficientes na incorporação de substâncias na superfície e também na porosidade de biomateriais de fosfato de cálcio [16, 19, 31]. Entretanto, o tipo de interação entre o fármaco e a matriz, se fisio ou quimiossorção, influencia tanto o processo de carregamento quanto o comportamento de liberação, in vivo ou in vitro $[16,17]$. Assim, são necessários estudos posteriores de liberação in vitro do sistema formado pela matriz bifásica de fosfato de cálcio-carboplatina produzida neste trabalho para melhor elucidar o tipo de interação formada.

\section{CONCLUSÕES}

Os resultados encontrados nesse estudo indicam que o método de alto vácuo foi eficiente na incorporação do fármaco na microestrutura microporosa do biomaterial bifásico micro e nanoestruturado 60\%HA/40\% TCP- $\beta$. Os dados obtidos mostraram claramente que o biomaterial bifásico granulado apresenta microestrutura microporosa interconectada formada por micro e nano grãos, favorável ao carregamento de fármacos. É importante frisar a necessidade da realização dos estudos de liberação da carboplatina em meio simulado, para depois, verificar as possibilidades de aplicações biomédicas em procedimentos oncológicos, no tratamento de tumores e reparação da estrutura óssea.

Os resultados encontrados são promissores e justificam a utilização da técnica em outros tipos de biocerâmicas microporosas de fosfatos de cálcio, utilizando doses diferenciadas. 


\section{AGRADECIMENTOS}

A CAPES pela bolsa de mestrado, ao CNPq pela bolsa PQ e a FAPESC pelo apoio financeiro.

\section{BIBLIOGRAFIA}

[1] ARCOS, D., VALLET-REGÍ, M. "Bioceramics for drug delivery", Acta Materialia, v. 61, pp. 890-911, 2013.

[2] CASTRO, S. S., ANDRADE, P. M. O., STONE, J. "Prevalência de deficiências motoras e sua relação com gastos federais com próteses, órteses e equipamentos nos estados brasileiros em 2010", Fisioterapia e Pesquisa, v. 22, n. 3, pp. 261-267, 2015.

[3] MARQUES, C., FERREIRA, J. M. F., ANDRONESCU, E., et al., "Multifuncional materials for bone cancer treatment", International Journal of Nanomedicine, v. 9, pp. 2713-2725, 2014.

[4] THIAN, E. S., HUANG, J., AHMAD, Z., et al., "Influence of nanohydroxyapatite patterns deposited by electrohydrodynamics spraying on osteoblast response", Journal of Biomedical Materials Research Part A, v. 85A, pp. 188-194, 2008.

[5] GHANAATI, S., BARBECK, M., ORTH, C., et al., "Influence of $\beta$-tricalcium phosphate granule size and morphology on tissue reaction in vivo", Acta Biomaterialia, v. 6, pp. 4476-4487, 2010.

[6] CAMARGO, N. H. A., FRANCZAK, P. F., GEMELLI, E., et al., "Characterization of three calcium phosphate microporous granulated bioceramics", Advanced Materials Research, v. 936, pp. 687-694, 2014.

[7] DALMÔNICO, G. M. L., FRANCZAK, P. F., LEVANDOWSKI, N., et al., "An in vivo study on bone formation behavior of microporous granular calcium phosphate", Biomaterials Science, pp. 1-11, 2017. DOI: 10.1039/c7bm00162b.

[8] SILVA, D. F., FRIIS, T. E., CAMARGO, N. H. A., et al., "Characterization of mesoporous calcium phosphate from calcareous marine sediments containing $\mathrm{Si}$, $\mathrm{Sr}$ and $\mathrm{Zn}$ for bone tissue engineering", Journal of Materials Chemistry B, v. 44, pp. 6842-6855, 2016.

[9] VERRON, E., KHAIROUN, I., GICHEUX, J., et al., "Calcium phosphate biomaterials as bone drug delivery systems: a review”, Drug Discovery Today, v. 15, n. 13/14, pp. 547-552, jul. 2010.

[10] BOSE, S., TARAFDER, S., EDGINGTON, J., et al., "Calcium phosphate ceramics in drug delivery", JOM., v. 63, n. 4, pp. 93-98, abr. 2011.

[11] VIGUIER, E., BIGNON, A., LAURENT, F., et al., "A new concept of gentamicin loaded HAP/TCP bone substitute for prophylatic action: in vivo pharmacokinetic study", Journal of Materials Science: Materials in Medicine, v. 22, pp. 879-886, 2011.

[12] VICTOR, S. P., KUMAR, T. S. S. "BCP ceramic microspheres as drug delivery carriers: synthesis, characterization and doxycycline release", Journal of Materials Science: Materials in Medicine, v. 19, pp. 283-290, 2008.

[13] JOSSE, S., FAUCHEUX, C., SOUEIDAN, A., et al., "Novel biomaterial for bisphosphonate delivery", Biomaterials. v. 26, pp. 2073-2080, 2005.

[14] SABER-SAMANDARI, S., NEZAFATI, N., SABER-SAMANDARI, S. "The effective role of hydroxyapatite-based composites in anticancer drug-delivery systems", Critical Reviews in Therapeutic Drug Carrier Systems, v. 33, n. 1, pp. 41-75, 2016.

[15] WATANABE, K., NISHIO, Y., MAKIURA, R., et al., "Paclitaxel-loaded hydroxyapatite/collagen hybrid gels as drug delivery system for metastatic cancer cells", International Journal of Pharmaceutics, v. 446, pp. 81-86, 2013.

[16] IAFISCO, M., MARGIOTTA, N. "Silica xerogels and hydroxyapatite nanocrystals for the local delivery of platinun-bisphosphonate complexes in the treatment for bone tumors: a mini-review", Journal of Inorganic Biochemistry, v. 117, pp. 237-247, 2012.

[17] PARENT, M., BARADARI, H., CHAMPION, E., et al., "Design of calcium phosphate for drug delivery application in bone desease: a review of the parameters affecting the loading and release of the therapeutic substance", Journal of Controlled Release, v. 252, pp. 1-17, 2017.

[18] NEVES, A. P., VARGAS, M. D. "Complexos de platina (II) na terapia do câncer", Revista Virtual de Química, v. 3, n. 3, pp. 196-209, 2011.

[19] COPATTI, C., CAMARGO, N. H. A., GEMELLI, E. "Incorporation of carboplatin in microporous granular calcium phosphate biphasic matrix”, Journal of Biosciences and Medicine, v. 2, pp. 30-35, 2014. 
[20] DALMÔNICO, G. M. L., SILVA, D. F., FRANCZAK, P. F., et al., "Elaboration biphasic calcium phosphate nanostructured powders", Boletin de la Sociedad Española de la Cerámica y Vidrio, v. 54, n. 1, pp. 37-43, 2015.

[21] CAMARGO, N. H. A., FRANCZAK, P. F., GEMELLI, E., et al., "Characterization of three calcium phosphate microporous granulated bioceramics”, Advanced Materials Research, v. 936, pp. 687-694, 2014.

[22] NIH US National Library of Medicine, PUB CHEM OPEN CHEMISTRY DATABASE, https://pubchem.ncbi.nlm.nih.gov/image/imagefly.cgi?cid=6251\&width=400\&height=400. Acessado em outubro de 2017.

[23] VANDERCANDELAERE, N., REY, C., DROUET, C. "Biomimetic apatite-based biomaterials: on the critical impact of synthesis and post-synthesis parameters", Journal of Materials Science: Materials in Medicine, v. 23, pp. 2593-2606, 2012.

[24] GÓMEZ-MORALES, J., IAFISCO, M., DELGADO-LÓPEZ, J. M., et al., "Progress on the preparation of nanocrystalline apatites and surface characterization: overview of fundamental and applied aspects", Progress in Crystal Growth and Characterization of Materials, v. 59, pp. 1-46, 2013.

[25] AZEVEDO, A. G. S., STRECKER, K., GORGULHO, H. F. "Efeito da temperatura em processos de sinterização de pós de hidroxiapatita", Cerâmica, v. 61, pp. 52-59, 2015.

[26] BRUNI, G., BERBENNI, V., MILANESE, C., et al., "Physico-chemical characterization of anhydrous D-mannitol", Journal of Thermal Analysis and Calorimetry, v. 95, n. 3, pp. 871-876, 2009.

[27] WYSOKINSKI, R., KUDUK-JAWORSKA, J., MICHALSKA, D. "Eletronic structure, Raman and infrared spectra, and vibrational assignment of carboplatin. Density functional theory studie", Journal of Molecular Structure: THEOCHEM, v. 758, pp. 169-179, 2006.

[28] LEVANDOWSKI JR., N., CAMARGO, N. H. A., SILVA, D. F., et al., "Characterization of different nanostructured bone substituted biomaterials", Advanced Materials Research, v. 936, pp. 695-700, 2014.

[29] CAMARGO, N. H. A., GEMELLI, E., MORAES, A. N., et al., "In vivo preliminary study on bone neoformation behavior of three types of calcium phosphate ceramics", Journal of Biosciences and Biomedicines, v. 2, pp. 36-42, 2014.

[30] COSTA, B. D., CAMARGO, N. H. A., OLESCOVICZ, N., et al., "Neoformação óssea e osteointegração de biomateriais micro e nanoestruturados em ovinos", Pesquisa Veterinária Brasileira, v. 35, n. 2, pp. 177-187, fev. 2015.

[31] ITOKAZU, M., ESAKI, M., YAMAMOTO, K., et al., "Local drug delivery system using ceramics: vacuum method for impregnating a chemotherapeutic agent into a porous hidroxyapatite block", Journal of Materials Science: Materials in Medicine, v. 10, pp. 249-252, 1999.

[32] GINEBRA, M. P., CANAL, C., ESPANOL, M., et al., "Calcium phosphate cements as drug delivery materials", Advanced Drug Delivery Reviews, v. 64, pp. 1090-1110, 2012.

\section{ORCID}

Cristiane Savicki

Nelson H A Camargo

Enori Gemelli

Cleomar Copatti

Nicolas A Camargo

Daiara Floriano Silva https://orcid.org/0000-0002-7323-7573

https://orcid.org/0000-0002-3330-2764

https://orcid.org/0000-0003-0881-4562

https://orcid.org/0000-0002-3791-0636

https://orcid.org/0000-0002-1335-3345

https://orcid.org/0000-0001-7964-6231 